\title{
Comparison of Robotic and Laparoscopic Partial Nephrectomy in Robotic Surgery Era
}

\author{
๑ Ekrem Güner, @ Selçuk Şahin \\ University of Health Sciences, Dr. Sadi Konuk Training and Research Hospital, Clinic of Urology, Istanbul, Turkey
}

\begin{abstract} bleeding, length of hospital stay and complications. min vs $16.3 \pm 7.3 \mathrm{~min}, \mathrm{p}=0.298$ ). be recommended to patients for early return to daily life.

Keywords: Partial nephrectomy, renal tumor, robotic surgery, laparoscopic surgery

\section{Introduction}

Nowadays, the development of radiological imaging methods and increasing accessibility have increased the incidence of incidentally detected renal masses. The majority of the detected masses are T1 tumors smaller than $7 \mathrm{~cm}$ and if technically feasible, it is recommended to remove only the mass by partial nephrectomy (1). Partial nephrectomy is traditionally performed with open technique, and can also be performed successfully with minimally invasive laparoscopic and robotic techniques. Minimally invasive techniques have been reported to offer shorter hospital stay, less blood loss and lower complication rates (2). Some studies have shown that robotic partial nephrectomy (RPN) reduces ischemia and suturing time even for an experienced laparoscopic surgeon.
\end{abstract}

Objective: To compare the results of robotic partial nephrectomy (RPN) and laparoscopic partial nephrectomy (LPN) operations and to determine whether they have any superiority to each other in terms of oncological and functional outcomes.

Materials and Methods: The data of patients who underwent partial nephrectomy due to renal tumor in our clinic were evaluated retrospectively. The data included demographic information, operative technique, tumor size, operative time, duration of warm ischemia, amount of intraoperative

Results: A total of 60 patients were included in the study. There was no significant difference between age (52.5 \pm 13.3 years vs $50.1 \pm 12.4$ years, $\mathrm{p}=0.48)$, body mass index ( $\left.26.9 \pm 3.7 \mathrm{vs} 27.3 \pm 3.3 \mathrm{~kg} / \mathrm{m}^{2}, \mathrm{p}=0.69\right)$ and tumor size $(3.2 \pm 1.4 \mathrm{~cm}$ vs $3.4 \pm 1.2 \mathrm{~cm}, \mathrm{p}=0.79)$ of patients who underwent LPN and RPN. The mean operative time $(194.5 \pm 44.6 \mathrm{~min}$ vs $203.3 \pm 22.2 \mathrm{~min}, \mathrm{p}<0.001)$ and length of hospitalization $(5 \pm 1.4$ days vs $6.2 \pm 2.1$ days, $\mathrm{p}=0.009)$ were significantly shorter in the RPN group. Although intraoperative bleeding was less in RPN patients, it was not statistically significant $(\mathrm{p}=0.065)$. Similarly, the duration of warm ischemia was lower in RPN patients than in LPN patients, but it was not statistically significant (14.3 \pm 7.8

Conclusion: RPN and LPN, which are minimally invasive surgical treatment options, can be used safely in the treatment of kidney tumors. RPN can

In this study, we aimed to compare the results of RPN and laparoscopic partial nephrectomy (LPN) operations in our clinic and to determine whether they have superiority in terms of oncologic and functional aspects.

\section{Materials and Methods}

After obtaining the approval of the Bakırköy Dr. Sadi Konuk Training and Research Hospital Ethics Committee (no: 2019/217), the data of patients who underwent partial nephrectomy due to renal tumor in the urology clinic of Bakırköy Dr. Sadi Konuk Training and Research Hospital between January 2015 and February 2019 were evaluated retrospectively. In patients undergoing LPN and RPN, renal hilar vascular control was performed by clamping the renal artery and vein separately. The operative time in RPN did not include the robot docking time.

Cite this article as: Güner E, Şahin S. Comparison of Robotic and Laparoscopic Partial Nephrectomy in Robotic Surgery Era. Bull Urooncol 2019;18(4):154-157

Address for Correspondence: Ekrem Güner, University of Health Sciences, Dr. Sadi Konuk Training and Research Hospital, Clinic of Urology, İstanbul, Turkey Phone: +90 2124147171 E-mail: ekremguner@yahoo.com ORCID-ID: orcid.org/0000-0002-4770-7535 
The data analyzed included demographic information, pre- and post-operative glomerular filtration rate (GFR) values, operative technique, tumor size, R.E.N.A.L. nephrometry score, operative time, duration of warm ischemia, amount of intraoperative bleeding, length of hospital stay and complications. Patients who were operated for benign pathologies and patients under 18 years of age were excluded from the study. The patients were divided into two groups as LPN group and RPN group. GFR was calculated using MDRD (Modification of diet in renal diseases study) formula $=186 \times(\text { Creatinine })^{-1.154} \times(\text { Age })^{-0.203} \times(0.742$ if female $) \times(1.210$ if black).

\section{Statistical Analysis}

IBM SPSS V.21 (Chicago, IL, USA) was used for statistical analysis of the data. Continuous variables were given as mean \pm standard deviation, while categorical variables were given as numbers and percentages. Kolmogorov-Smirnov test was used to test the normality of data. For pairwise comparison, Student's t-test and Mann-Whitney $U$ test were used for numerical data and chi-square test was used for categorical variables. $\mathrm{P}<0.05$ was considered statistically significant.

\section{Results}

A total of 60 patients ( 38 male, 22 female) were included in the study. The mean age of the patients was $51.3 \pm 12.8$ years. The mean tumor size was $3.3 \pm 1.3 \mathrm{~cm}$. There were 30 consecutive patients whose data were available for LPN and RPN groups (Table 1). There was no significant difference between mean age (52.5 \pm 13.3 years vs $50.1 \pm 12.4$ years, $p=0.48)$, body mass index $\left(26.9 \pm 3.7\right.$ vs $\left.27.3 \pm 3.3 \mathrm{~kg} / \mathrm{m}^{2}, \mathrm{p}=0.69\right)$ and tumor size $(3.2 \pm 1.4$ $\mathrm{cm}$ vs $3.4 \pm 1.2 \mathrm{~cm}, \mathrm{p}=0.79$ ) of patients who underwent LPN and RPN. The mean R.E.N.A.L. score of both groups were similar $(p=0.642)$. ASA scores were similar for both groups $(p=0.254)$. The mean operative time (194.5 $\pm 44.6 \mathrm{~min}$ vs $230.3 \pm 22.2 \mathrm{~min}$, $\mathrm{p}<0.001)$ and length of hospital stay $(5 \pm 1.4$ days vs $6.2 \pm 2.1$ days, $p=0.009$ ) were significantly shorter in the RPN group. Although the amount of intraoperative bleeding was less in RPN patients, it was not statistically significant $(206 \pm 94.4 \mathrm{~mL}$ vs $246.8 \pm 72.2 \mathrm{~mL}, \mathrm{p}=0.065$ ). Similarly, the duration of warm ischemia was lower in RPN patients than in LPN patients but it did not reach statistical significance $(14.3 \pm 7.8 \mathrm{~min}$ vs $16.3 \pm 7.3 \mathrm{~min}, \mathrm{p}=0.298$ ). Post-operative complication rates and characteristics were similar in LPN and RPN groups according

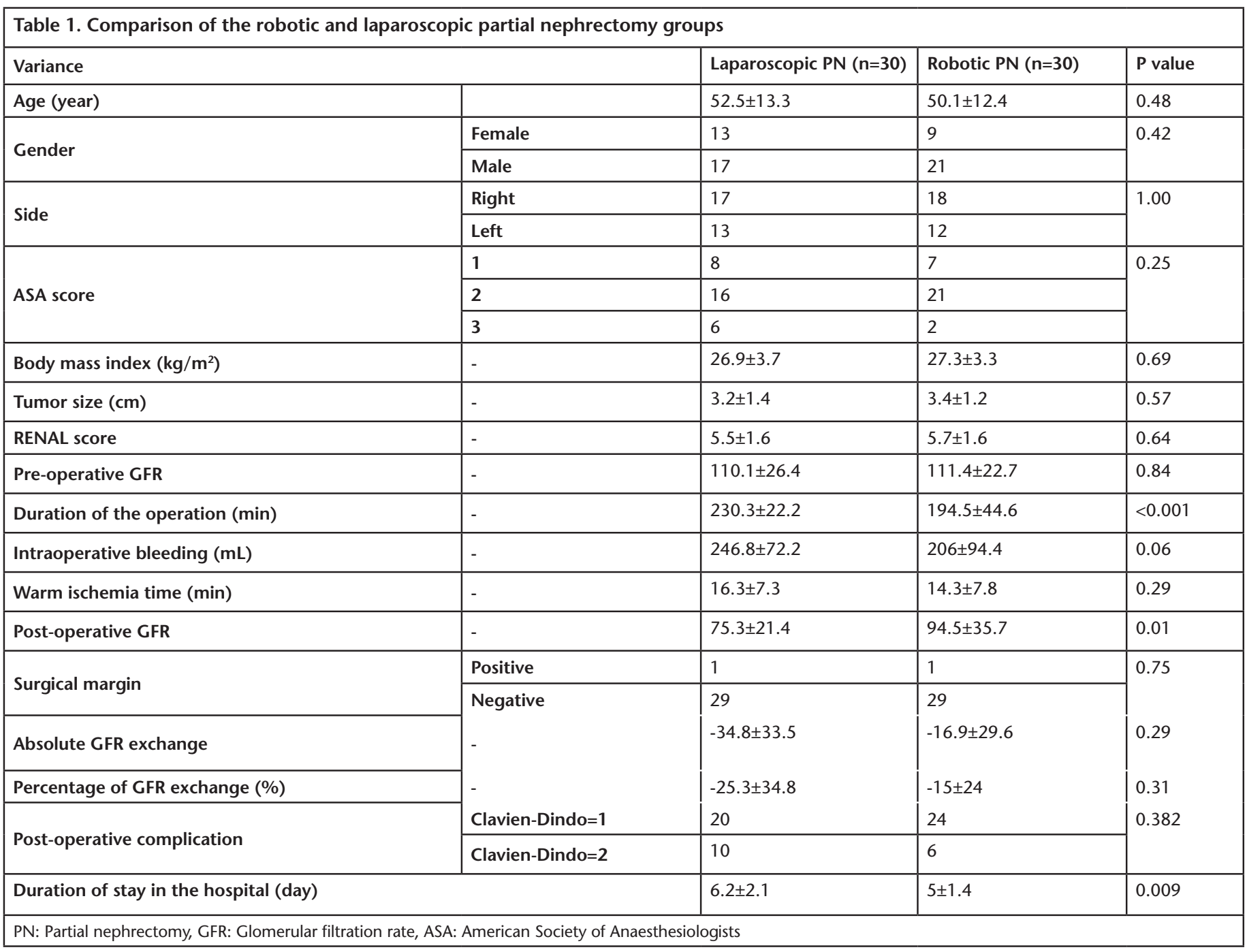


to Clavien-Dindo classification ( $p=0.382)$. Surgical margin positivity was detected in one patient (3.3\%) in both patient groups. Patients with positive surgical margins were followed up conservatively and no recurrence was observed at a mean follow-up of 18 months. The decrease in GFR was higher in the LPN group in both units and percent $(p=0.029$ and $p=0.031$, respectively).

\section{Discussion}

Partial nephrectomy is the gold standard in the treatment of renal tumors, especially those smaller than $4 \mathrm{~cm}$ (3). However, it is recommended to remove larger renal masses by partial nephrectomy when technically possible. Over the years, partial nephrectomy has evolved from open to minimally invasive laparoscopic and finally to robotic techniques. In many different studies, RPN has been shown to be superior to LPN in various aspects (4). In this study, we demonstrated that RPN is superior to LPN in some aspects, but they are similar in terms of oncologic outcomes and preservation of renal function.

In a multicenter prospective study by Alimi et al. (5), shortterm oncologic and functional results of RPN and LPN were found to be similar. According to this study, LPN was associated with longer warm ischemia ( $23 \mathrm{~min}$ vs $15.7 \mathrm{~min}$ ) and longer hospital stay (4.6 days vs 3.6 days), whereas intraoperative blood loss was higher in RPN ( $381 \mathrm{~mL}$ vs $215 \mathrm{~mL}$ ). Perioperative complications and positive surgical margin rates were reported to be similar in RPN (2\%) and LPN (6\%) groups (5). In another study by matching the patients according to nephrometry scores, RPN operative time was found to be shorter than LPN in all nephrometry scores, whereas ischemia time and hospital stay were lower in nephrometry scores greater than 7 (2). In another study conducted by Faria et al. (6), RPN was found to be superior to LPN in terms of renal function, warm ischemia time, suture time, renorrhaphy time $(p<0.05)$. In another study by Kim et al. (7), similarly, operative and warm ischemia times were found to be shorter in RPN patients than in LPN. In addition, recovery of renal function after partial nephrectomy has been reported to be better in RPN patients. In our study, RPN was superior to LPN in terms of operative time and hospital stay. Although the amount of bleeding and warm ischemia time was lower in RPN patients, no statistical significance was observed. However, absolute and percent GFR reduction was significantly higher in LPN patients than in RPN patients. This difference may be related to the amount of bleeding or ischemia time.

In a multicenter study by Zargar et al. (8), RPN and LPN were compared in terms of trifecta (negative surgical margin, zero perioperative complications, and duration of warm ischemia less than $25 \mathrm{~min}$ ). In the study, $1185 \mathrm{RPN}$ and $646 \mathrm{LPN}$ patients were included, and the rate of achieving trifecta was $70 \%$ in RPN and $33 \%$ in LPN. In a meta-analysis by Choi et al. (3) comparing RPN to LPN, no difference was found between the two types of operations in terms of complication rates, serum creatinine change, operative time, estimated blood loss and surgical margin positivity according to Clavien-Dindo classification. However, less switching to open surgery or radical surgery has been reported in RPN. In addition, the duration of warm ischemia and hospitalization were lower in RPN patients. The surgical margin positivity and blood loss data in our study were also in line with the mentioned studies. There was no significant difference between the complication rates according to Clavien-Dindo classification.

When comparing LPN and RPN operations and recommending them to patients, it would be appropriate to perform costeffectiveness analysis. Even in a study in the United States, the cost of RPN was $\$ 1066$ more than LPN per case (9). The cost of maintenance of the robotic system as well as the purchase costs should be well analyzed for developing countries like us.

\section{Study Limitations}

Our study has, of course, limitations. First of all, this study was performed retrospectively in a single center. Failure to randomize patients may have led to bias during patient selection. Although all of the surgeons were experienced in the field, different surgeons may also have an impact on the results.

\section{Conclusion}

RPN and LPN, which are minimally invasive surgical treatment options, can be used safely in the treatment of renal tumors. RPN can be recommended to patients for better preservation of renal reserve and early return to daily life.

\section{Ethics}

Ethics Committee Approval: This study is approved by the Bakırköy Dr. Sadi Konuk Training and Research Hospital Ethics Committee (no: 2019/217).

Informed Consent: Due to this study is retrospective, informed consent had not obtained.

Peer-review: Externally peer-reviewed.

\section{Authorship Contributions}

Concept: E.G., S.Ş, Design: E.G., S.Ş, Data Collection or Processing: E.G., S.Ş, Analysis or Interpretation: E.G., S.Ş, Literature Search: E.G., S.Ş, Writing: E.G., S.Ş.

\section{Acknowledgements}

Publication: The results of the study were not published in full or in part in form of abstracts.

Contribution: There is not any other contributors who may not be listed as authors.

Conflict of Interest: No conflict of interest was declared by the authors.

Financial Disclosure: The authors declared that this study received no financial support.

\section{References}

1. Laviana AA, Hu JC. Current controversies and challenges in roboticassisted, laparoscopic, and open partial nephrectomies. World J Urol 2014;32:591-596.

2. Banapour P, Abdelsayed GA, Bider-Canfield Z, et al. Nephrometry score matched robotic vs. laparoscopic vs. open partial nephrectomy. J Robot Surg 2018;12:679-685.

3. Choi JE, You JH, Kim DK, et al. Comparison of perioperative outcomes between robotic and laparoscopic partial nephrectomy: a systematic review and meta-analysis. Eur Urol 2015;67:891-901. 
4. Luciani LG, Chiodini S, Mattevi D, et al. Robotic-assisted partial nephrectomy provides better operative outcomes as compared to the laparoscopic and open approaches: results from a prospective cohort study. J Robot Surg 2017;11:333-339.

5. Alimi Q, Peyronnet B, Sebe P, et al. Comparison of Short-Term Functional, Oncological, and Perioperative Outcomes Between Laparoscopic and Robotic Partial Nephrectomy Beyond the Learning Curve. J Laparoendosc Adv Surg Tech A 2018;28:1047-1052.

6. Faria EF, Caputo PA, Wood CG, et al. Robotic partial nephrectomy shortens warm ischemia time, reducing suturing time kinetics even for an experienced laparoscopic surgeon: a comparative analysis. World J Urol 2014;32:265-271.
7. Kim JH, Park YH, Kim Y], et al. Perioperative and long-term renal functional outcomes of robotic versus laparoscopic partial nephrectomy: a multicenter matched-pair comparison. World J Urol 2015;33:1579-1584.

8. Zargar H, Allaf ME, Bhayani S, et al. Trifecta and optimal perioperative outcomes of robotic and laparoscopic partial nephrectomy in surgical treatment of small renal masses: a multi-institutional study. BJU Int 2015;116:407-414.

9. Hyams E, Pierorazio P, Mullins JK, et al. A comparative cost analysis of robot-assisted versus traditional laparoscopic partial nephrectomy. J Endourol 2012;26:843-847. 